\title{
Characterization of Intestinal Dendritic Cells in Murine Norovirus Infection
}

\author{
Xiuxu Chen, Daniel Leach, Desiré A. Hunter, Daniel Sanfelippo, Erika J. Buell, Sarah J. Zemple \\ and Mitchell H. Grayson*
}

Division of Allergy and Clinical Immunology, Department of Pediatrics, Medical College of Wisconsin, Milwaukee, WI 53226, USA

\begin{abstract}
We have shown that respiratory viral infections drive allergic disease through dendritic cells, whether gastrointestinal viruses induce allergies are not known. Norovirus infections are a major cause of gastroenteritis in humans. We used murine norovirus (MNV) to explore the effect of MNV infection on gastrointestinal conventional DCs (cDCs) and plasmacytoid DCs (pDCs). MNV infection induced disparate effects on cDCs and pDCs in lymphoid tissues of the small intestine and draining mesenteric lymph nodes. FceRI was transiently expressed on lamina propria cDCs, but not on pDCs. In addition, feeding ovalbumin during the viral infection led to a modest, brief induction of anti-ovalbumin IgE. Together, these data suggest that like with a respiratory viral infection, an intestinal viral infection may be sufficient to induce changes in DCs and the generation of food-specific IgE. Whether this represents a novel mechanism of food allergy remains to be determined.
\end{abstract}

Keywords: Allergy, dendritic cells, gastrointestinal infection, IgE, immune response, norovirus, virus.

\section{INTRODUCTION}

Paramyxoviral and Orthomyxoviral respiratory viral infections have been shown to increase the risk of developing allergic disease [1-5]. We have shown that the murine paramyxovirus, Sendai Virus $(\mathrm{SeV})$, in a mouse model, causes mucous cell metaplasia that critically depends upon the function of dendritic cells $[6,7]$. Our studies and those of others have shown that dendritic cells are critical for the translation of Th1-dominant anti-viral immune responses into a Th2-skewed allergic response $[5,6]$. In the case of $\mathrm{SeV}$ infection, crosslinking of the high affinity $\mathrm{IgE}$ receptor (FceRI) on conventional dendritic cells (cDC) led to production of CC chemokine ligand 28 (CCL28), which in turn attracted interleukin 13 (IL-13) producing Th2 cells into the airways, driving the pro-atopic response [6].

Intriguingly, paramyxoviruses and orthomyxoviruses, such as Respiratory syncytial virus (RSV), SeV and Influenza A are all negative-sense, single-stranded RNA viruses. It remains unclear whether this effect is specific to these viruses or is a general feature for other single-stranded RNA viruses, such as norovirus. Human norovirus is a major gastrointestinal pathogen and accounts for over $90 \%$ of all nonbacterial epidemic gastroenteritis, causing nearly 23 million infections, around 50 thousand hospitalizations, and 300 deaths annually [8-11]. Development of a vaccine and long-term immunity to the human norovirus has not been achieved, mainly due to highly diverse strain variation and lack of cross-protection amongst various strains [10]. Recently a mouse norovirus (MNV) was identified [12]. Previous studies have shown that the STAT-1 signaling

*Address correspondence to this author at the Medical College of Wisconsin, MFRC Room 5064, 8701 Watertown Plank Road, Milwaukee, WI 53226, USA; Tel: 414-456-5648; Fax: 414-456-6323;

E-mail: wheeze@allergist.com pathway is essential for mice survival from oral, intranasal, or intracranial challenge with MNV. Further, multiple deficiencies of type I and II interferon (IFN) receptors or perforin lead to increased susceptibility to viral infection with MNV [12, 13]. However, the role that gastrointestinal dendritic cells play in the anti-viral response to this virus is unknown.

Dendritic cells straddle the innate and adaptive immune system and play a crucial role in controlling immunity to foreign invasion and regulating responses to self-antigens. Dendritic cells are a functionally heterogeneous population that have been classified into two main subtypes in the mouse: conventional dendritic cells (cDC), which express the $\beta 2$ integrin CD11c (cDCs), and plasmacytoid dendritic cells (pDC), which express PDCA-1 and Siglec H [14-16].

cDCs have further been divided into different subsets based on various surface markers, most commonly CD11b, CD8 $\alpha$, CD103, CX3CR1, and CD4 depending on the tissue analyzed and immune response [17]. Earlier studies suggested that these dendritic cell populations have marked functional differences. For example, CD $8 \alpha^{+} \mathrm{cDCs}$ were felt to be more biased to differentiate naïve T cells into IFN- $\gamma$ secreting Th1 cells, while $\mathrm{CD} 11 \mathrm{~b}^{+} \mathrm{cDCs}$ were felt to drive a more tolerogenic response $[18,19]$. This is the opposite in humans, and $\mathrm{CD} 03^{+} \mathrm{cDCs}$, particularly from the lamina propria, appear immunotolerogenic via retinoic acidmediated transforming growth factor- $\beta$ (TGF- $\beta$ ) production, as well as being required for immune homeostasis through induction of regulatory $\mathrm{T}$ cells [20-23]. However, this is likely too simplistic, as recent studies have demonstrated that $\mathrm{CD} 8 \alpha^{+}$and $\mathrm{CD} 103^{+} \mathrm{cDCs}$ are similar in terms of their transcriptome and dependence on Interferon Regulatory Factor 8 [24]. Compared to $\mathrm{cDC}$, pDCs have been relatively under-studied, despite the fact that they were first reported as $\mathrm{T}$ cell-associated plasma cells or plasmacytoid $\mathrm{T}$ cells nearly twenty years ago [25]. These cells are characterized by their 
ability to produce large amounts of type I IFN during a viral infection [26-28]. Due to heterogeneous expression of CD4 and $\mathrm{CD} 8 \alpha$ on pDCs, these cells can be divided into subsets [29]. However, even though studies have reported additional pDC subsets, when compared with cDCs the pDCs appear much more homogeneous [30, 31]. Whether any of these subsets have functional differences remains to be determined.

We undertook this study to characterize gastrointestinal dendritic cells in response to a murine norovirus (MNV) infection. Our data demonstrate differential kinetics in the accumulation of $\mathrm{cDCs}$ and $\mathrm{pDCs}$ in mouse small intestine lamina propria. Intriguingly and similar to what we have reported in the lung, MNV infection was found to induce FceRI expression on lamina propria cDCs, and IgE against an orally administered protein. Together these data suggest that infection with this Caliciviridae family member might play a role in development of allergy - and in particular, food allergy - similar to what we have shown for $\mathrm{SeV}[6,32]$.

\section{MATERIALS AND METHODOLOGY}

\section{Ethics Statement}

All procedures performed on mice were in accordance with the NIH guidelines for humane treatment of animals and were approved by the Medical College of Wisconsin's Institutional Animal Care and Use Committee (IACUC). The IACUC approved protocol number is AUA764.

\section{Reagents}

The following reagents were used: Isoflurane (NDC 57319-507-06, Phoenix Pharmaceutical, Inc. St. Joseph MO), collagenase I (Worthington Biochemical Corporation, Lakewood NK), hyaluronidase type II (Sigma-Aldrich, St. Louis MO), DNase I (Worthington Biochemical), EDTA (AMRESCO Inc, Solon OH), 4',6-diamidino-2-phenylindole (DAPI, Thermo Scientific, Rockford IL), ovalbumin (OVA, Sigma-Aldrich), Tissue-Tek OCT compound (Sakura Finetek USA, Inc. Torrance CA), paraformaldehyde (SigmaAldrich); Fluoromount G (EMS, Hatfield PA); Trizol (Invitrogen Corporation, Carlsbad CA).

Phytoerythrin-, allophycocyanin-, FITC-, or Alexa Fluor 647-labeled Abs against mouse CD11c (clone N418), FceRI $\alpha$ (clone MAR-1), CD23 (clone B3B4), CD86 (clone GL1), CD103 (clone M290), CD11b (clone M1/70), CD4 (clone RM4-5), CD8 $\alpha$ (clone 53-6.7), CD3 (clone 17A2), MHC class II (clone M5/114.15.2), and isotype control IgGs were obtained from eBioscience (San Diego, CA), BD Pharmingen (San Diego, CA), and/or Biolegend (San Diego, CA). Rat anti-mouse PDCA-1 was from Miltenyi Biotec (Auburn CA).

\section{Mice}

C57BL/6J mice were from The Jackson Laboratory (Bar Harbor, ME). Mice were housed, handled, and experiments performed according to protocols approved by the Institutional Animal Care and Use Committee at the Medical College of Wisconsin (Milwaukee, WI).

\section{Preparation of MNV-CW1 Virus Stock}

Murine norovirus (strain MNV-CW1) and mouse macrophage cell line Raw 264.7 were kindly provided by Dr. Herbert W. Virgin (Washington University, St. Louis, MO) and Dr. John Routes (Medical College of Wisconsin, Milwaukee, WI), respectively. Raw 264.7 cells were cultured in complete Dulbecco's Modified Eagle Medium (DMEM; 2 $\mathrm{mM}$ L-glutamine; $100 \mu \mathrm{g} / \mathrm{ml}$ each of penicillin and streptomycin (Invitrogen, Carlsbad, CA); 10\% FBS (Atlanta Biologicals, Lawrenceville, GA)) and were incubated with MNV-CW1 at 0.05 multiplicity of infection (MOI) in $75 \mathrm{~cm}^{2}$ flasks until 50-80\% cytopathic effect (CPE). Cells were harvested by scraping followed by freeze-thawing to release virus. Viral stocks were prepared by an initial low speed centrifugation to remove cell debris, followed by ultracentrifugation at 27,000 rpm for 3 hours. Viral titer was determined by standard plaque assay using RAW 264.7 cells in 6-well plates at $0.05 \mathrm{MOI}$ and $\sim 80 \% \mathrm{CPE}$. Viral stocks were stored at $-80^{\circ} \mathrm{C}$. Ultraviolet light inactivated $\mathrm{MNV}$ (UV-MNV) was made by exposing the diluted aliquot of MNV to 240,000 $\mu \mathrm{J}$ of UV light (Stratalinker UV Crosslinker 1800; Stratagene, La Jolla, CA). The effectiveness of UV inactivation was verified by the inability of UV-MNV to replicate in RAW 264.7 cells (data not shown).

\section{Preparation of Tissue Cells}

Following euthanasia, the mesenteric lymph node (MLN) was removed, and then the small intestine was removed and sliced longitudinally. The intestinal tissue was then rinsed at least 3 times with $4^{\circ} \mathrm{C}$ phosphate buffered saline (PBS) and all visible Peyer's Patches (PP) and any additional mesenteric tissue removed. The remaining intestinal tissue was used as the source of the lamina propria (LP) cells, as mentioned in reference [20]. The PP, MLN, or LP were separately cut into small pieces in a petri dish with $4 \mathrm{ml}$ prewarmed digestion buffer (collagenase I, $50 \mu \mathrm{g} / \mathrm{ml}$ DNase I, and hyaluronidase type II in DMEM as described [16]), and incubated at $37^{\circ} \mathrm{C}$ in $5 \% \mathrm{CO}_{2}$ incubator. During digestion, the petri dishes were gently agitated every 10-15 minutes. After $45 \mathrm{~min} ., 1 \mathrm{ml}$ of $10 \mathrm{mM}$ ethylenediaminetetraacetic acid (EDTA) was added and cells were cultured for another 15 minutes. A single cell suspension was obtained by filtering the culture fluid through a $70 \mu \mathrm{m}$ cell strainer. Tissue clumps were discarded and the cell suspension washed with DMEM twice before being used for antibody staining and flow cytometric analysis. In some experiments, LP-cDC were isolated using CD11c microbeads (Miltenyi Biotec, Auburn, CA) as previously described [7]. These cells were then cultured at $10^{5}$ cells $/ \mathrm{ml}$ in DMEM with $10 \%$ fetal calf sera with either MNV (at $0.10 \mathrm{MOI}$ ) or PBS for 24 hours at $37^{\circ} \mathrm{C}$ with $5 \% \mathrm{CO}_{2}$. After culture, cell death was assayed by flow cytometry using propidium iodide (BioLegend, San Diego, CA) exclusion.

\section{Fluorescence Microscopy}

Sixteen hours after MNV-CW1 infection, mice were euthanized, and the proximal, middle, and distal $1 / 3$ of the small intestine was collected and embedded in blocks with Tissue-Tek OCT compound and frozen at $-80^{\circ} \mathrm{C}$ for cryosectioning. Five $\mu \mathrm{m}$ sections were obtained and air-dried before being stored at $-80^{\circ} \mathrm{C}$. For staining, slides were warmed to room temperature and then nonspecific binding blocked by adding excess rat anti-mouse IgG Fc-specific blocking antibody (clone 2.4G2) for 30 minutes at $4{ }^{\circ} \mathrm{C}$. Slides were stained with DAPI and $5 \mu \mathrm{g} / \mathrm{ml}$ anti- CD11c-PE or isotype control IgG-PE for 30 minutes at $4^{\circ} \mathrm{C}$ in the dark. 
After rinsing three times in $1 \%$ bovine serum albumin (BSA) in PBS (PBS/BSA), slides were fixed for 10 minutes with $2 \%$ paraformaldehyde at room temperature. After washing with PBS/BSA, mounting medium was added and the slides covered with cover slips, which were sealed with nail polish. Pictures were acquired on Nikon eclipse E400 (Nikon Inc, Melville NY) and analyzed with Zeiss LSM software.

\section{Flow Cytometry}

Samples were stained with the indicated specific monoclonal antibodies (mAbs) or negative control mAbs in FACS buffer (PBS/BSA containing $2 \mu \mathrm{g} / \mathrm{ml} 4$ ',6-diamidino2-phenylindole (DAPI)) for $30 \mathrm{~min}$ on ice, before being washed and re-suspended in FACS buffer without DAPI. Data were collected on an LSR II flow cytometer (Becton Dickinson, Franklin Lakes, NJ) and analyzed using Flowjo software (Tree Star, Ashland, OR). Live cells were identified based on forward and side scatter as well as exclusion of DAPI. For cell sorting, cells were first stained with $2 \mu \mathrm{g} / \mathrm{ml}$ of the appropriate mAb (anti-CD11c, anti-PDCA-1, antiCD4, or appropriate isotype control Abs) and sorted on a FACSAria cell sorter (Becton Dickinson).

\section{Oral Gavage}

Using a gavage needle (Popper, New Hyde Park, NY) $120 \mu \mathrm{L}$ of MNV, UV-MNV, PBS, or $1 \mathrm{mg} / \mathrm{ml}$ OVA (in PBS) were injected into the mouse's stomach. The gavage needle was attached to a $1 \mathrm{cc}$ syringe and was inserted in the mouth along the back/dorsal aspect of the throat, beginning at approximately $45^{\circ}$ angle and then gradually becoming vertical. The syringe plunger was then depressed. The syringe and needle were then rapidly removed.

\section{Enzyme-Linked Immunosorbent Assay (ELISA)}

A standard ELISA for anti-OVA IgE was performed as previously described [32]. Briefly, 96-well Maxi Sorp (Nalge Nunc) plates were coated over night with OVA. After being washed, mouse sera was added to the wells, and OVA specific IgE was determined using a detection anti-IgE antibody (BD Biosciences Pharmingen, San Diego, California). Because of variability in the baseline values, the optical density (OD) at $450 \mathrm{~nm}$ for each animal's day 7 sample (day 12 post MNV or UV-MNV) was normalized to the value seen before administration of OVA (day 5 post MNV or UV-MNV) for that animal.

\section{Statistical Analysis}

Unless otherwise stated, all data are presented as mean \pm sem. Two-sided unpaired Student's $t$ test was used for all parametric data, with $p<0.05$ considered significant.

\section{RESULTS} The Frequency of Lamina Propria Conventional Dendritic
Cells (cDCs) is Reduced with MNV Infection

To investigate the role of dendritic cells in an MNV infection, we first analyzed the frequency of $\mathrm{cDC}$ in the lamina propria of the small intestine (LP-cDCs). C57BL/6 mice were given $5 \times 10^{5} p f u$ MNV-CW1 (MNV), ultraviolet- inactivated MNV-CW1 (UV-MNV), or PBS vehicle control by gavage, and LP-cDCs in different segments of small intestine were analyzed microscopically 16 hours post inoculation (p.i.). We observed that $\mathrm{CD} 11 \mathrm{c}^{+} \mathrm{cDCs}$ are present primarily in the lamina propria tissue, with very few being noted intraepithelially (Fig. 1). The frequency of CD11 ${ }^{+}$LP-cDCs in the villi of the small intestine was reduced in MNV infected mice compared with UV-MNV or PBS treated mice. Interestingly, reduction of $\mathrm{CD} 11 \mathrm{c}^{+}$cells ranged in a longitudinal pattern from the proximal to the distal portion of the small intestine, with the most prominent reduction in the proximal $1 / 3$ portion of the small intestine (Fig. 1).

\section{MNV Infection Induces Tissue Department Specific Kinetics for cDCs}

Based on the above histological observation, we next determined the effect of MNV on the frequency and phenotype of cDCs from different lymphoid tissues. In particular, we analyzed LP-cDCs, Peyer's Patch-cDCs (PP-cDCs) and mesenteric lymph node-cDCs (MLN-cDCs) by flow cytometry. To isolate $\mathrm{CD} 11 \mathrm{c}^{+} \mathrm{cDCs}$, we initially gated on cellular events using forward and side scatter, using 4',6-diamidino-2phenylindole (DAPI) to exclude non-specific staining from cell death, and calculated the frequency of $\mathrm{CD} 11 \mathrm{c}^{+}$cells in the $\mathrm{DAPI}^{\text {low }}$ gate (Fig. 2A). The resulting kinetics of $\mathrm{cDC}$ accumulation post MNV inoculation are shown for the LP, PP, and MLN (Fig. 2B). In corroboration with our histologic data, we found that the frequency of LP-cDCs significantly decreased 1 day p.i. MNV, and remained suppressed through day 3 p.i. (Fig. 2B). In both of the lymphoid organs associated with the intestinal tract, the MLN and PP, there was an insignificant drop in $\mathrm{CDC}$ at 1 day p.i. with a return to baseline values by day 3 p.i. (Fig. 2B). In all cases, UV-MNV or PBS treatment failed to significantly alter the frequency of any CDC population from their pre-inoculation levels (Fig. 2B). To determine if MNV induced cell death of LP-cDC, we cultured MNV (at 0.10 MOI) with LP-cDC. After 24 hours we found a non-significant increase in LP-cDC death with MNV $(67.4 \pm 13.6 \%$ dead, $n=4)$ when compared to PBS control ( $30.4 \pm 30 \%$ dead, $\mathrm{n}=2, \mathrm{p}=0.25)$.

\section{Differential Effect of MNV Infection on cDC Subsets}

In the lung, different subsets of dendritic cells are known to play different roles during the course of influenza infection [33]. We next studied whether the composition of cDCs in the LP, MLN and PP were affected. For these studies, we analyzed four surface markers, CD103, CD11b, $\mathrm{CD} 4$ and $\mathrm{CD} 8 \alpha$, which have previously been reported to identify distinct subsets of mouse cDCs.

In the LP the vast majority of $\mathrm{CD} 11 \mathrm{c}^{+} \mathrm{cDC}$ expressed $\mathrm{CD} 11 \mathrm{~b}$, and this was unaffected by MNV inoculation suggesting that the decrease in $\mathrm{CDC}$ seen with MNV inoculation is equally spread over all subsets of cDC (Fig. 2C). In the MLN we found that the major $\mathrm{cDC}$ subsets were CD11b and CD103 expressing cells, and upon active viral infection (MNV) there was a significant and selective decrease in the $\mathrm{CD} 103^{+} \mathrm{cDC}$ population (Fig. 2C), although the absolute change remained quite modest. 

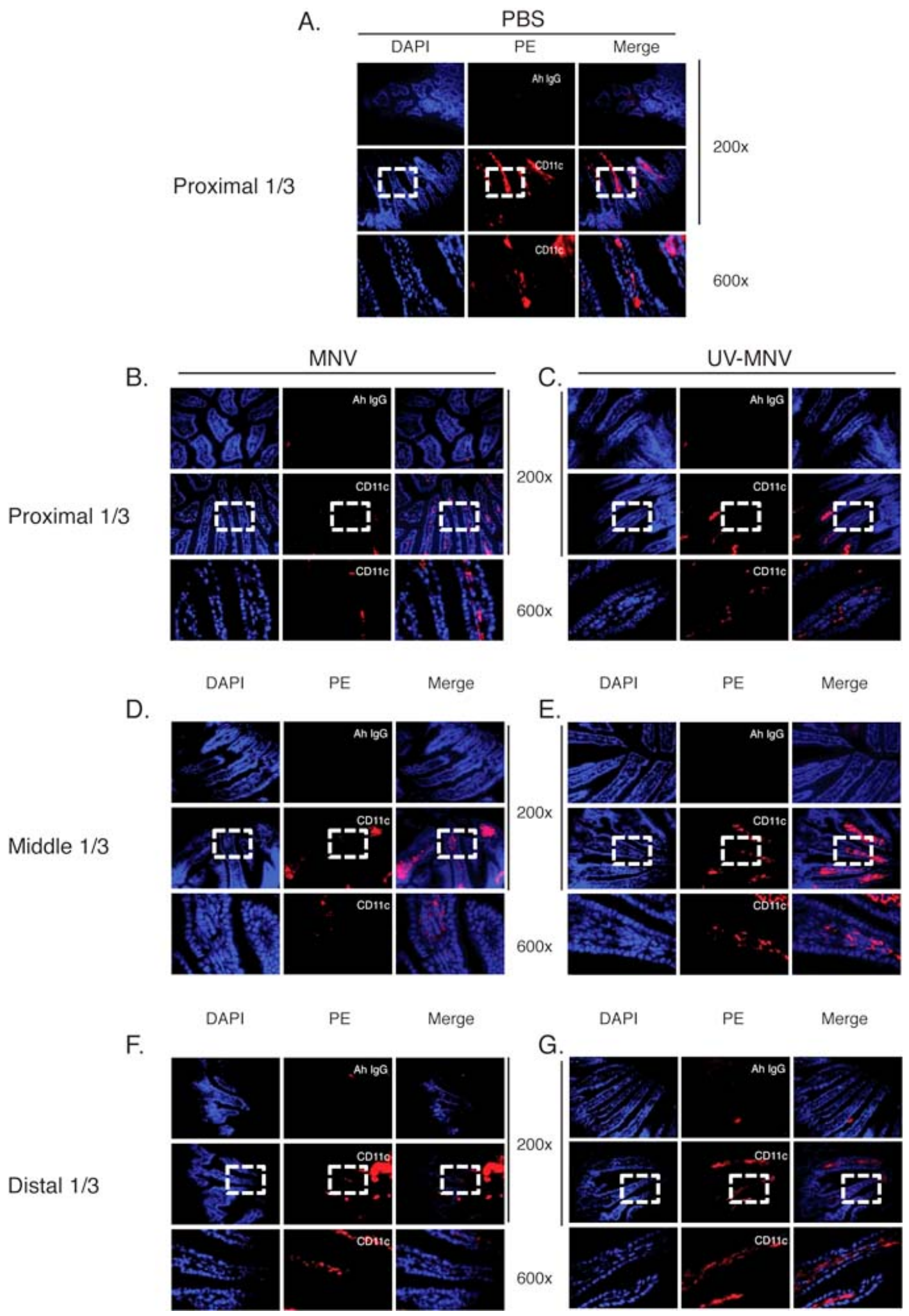

Fig. (1). The frequency of lamina propria conventional dendritic cells (LP-cDC) is reduced after MNV infection. LP-cDC were identified by CD11c expression in the lamina propria of frozen sections from small intestine of C57BL/6 mice. Mice were inoculated by oral gavage with PBS (A), MNV-CW1 (MNV; $5 \times 10^{5}$ pfu per mouse) (B, D, F), or ultraviolet light-inactivated MNV (UV-MNV) (C, E, G). Sixteen hours post inoculation (p.i.), mice were sacrificed and cryosections from the proximal 1/3 (A, B, C), middle 1/3 (D, E) and distal 1/3 $(\mathbf{F}, \mathbf{G})$ of the small intestine were stained with PE-labeled rat anti-mouse CD11c monoclonal antibody or isotype rat IgG control (red) and DAPI (blue), and visualized by epi-fluorescence microscope. Frequency of CD11 c LP-cDCs in the vili of the small intestine was analyzed by Zeiss LSM software. Representative images from three independent experiments of 2 mice per experiment are shown. Ah IgG = Armenian hamster IgG control. 
A.

Lamina propria
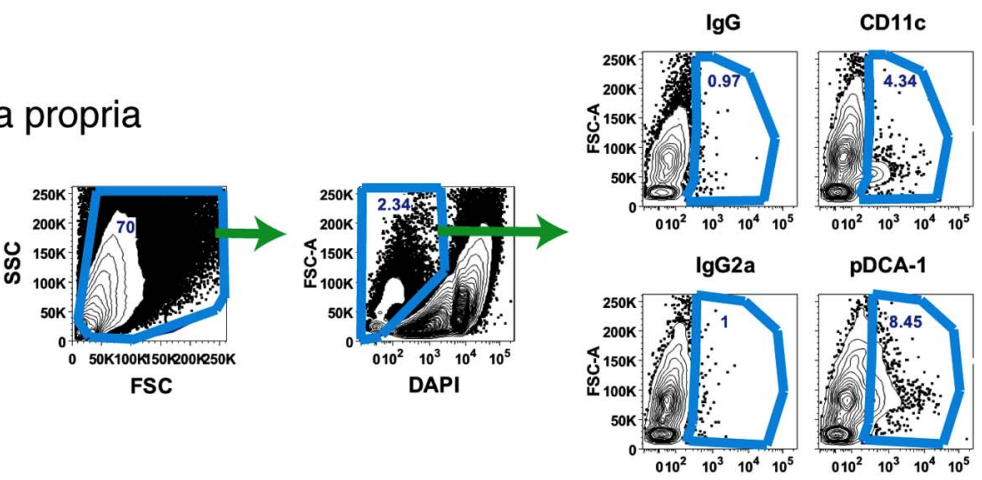

CD11c

Peyer's patches and Mesenteric lymph node

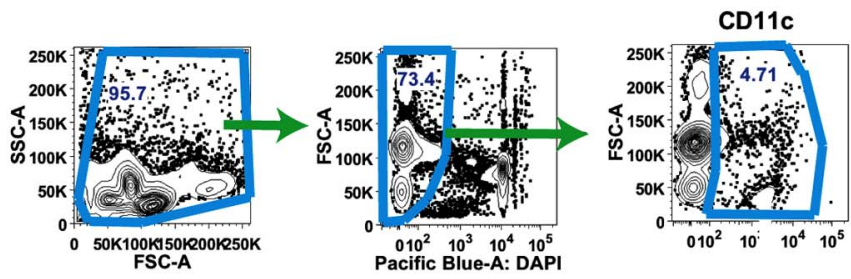

B.
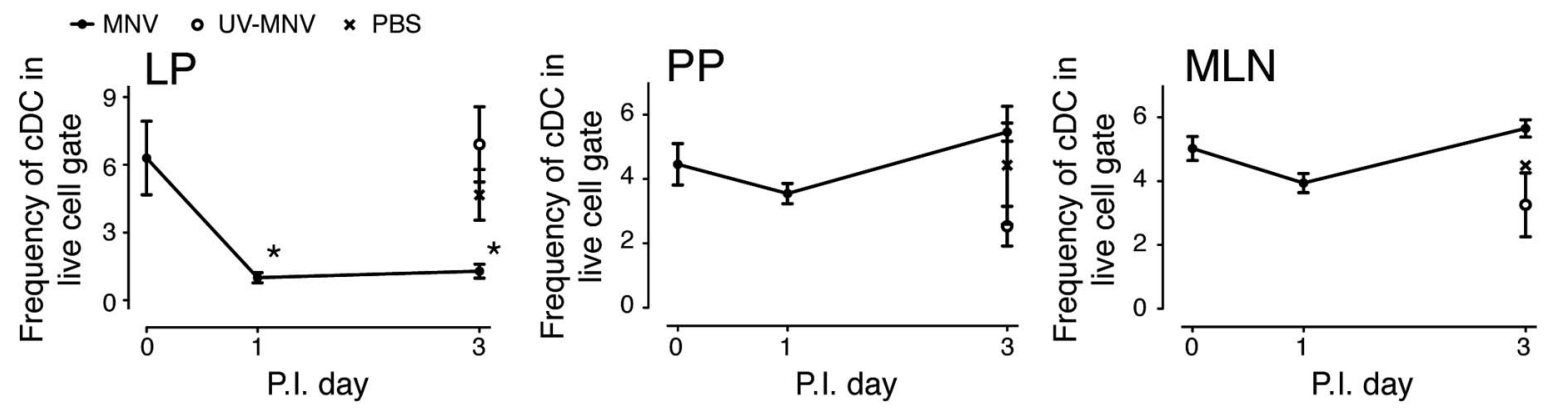

C.
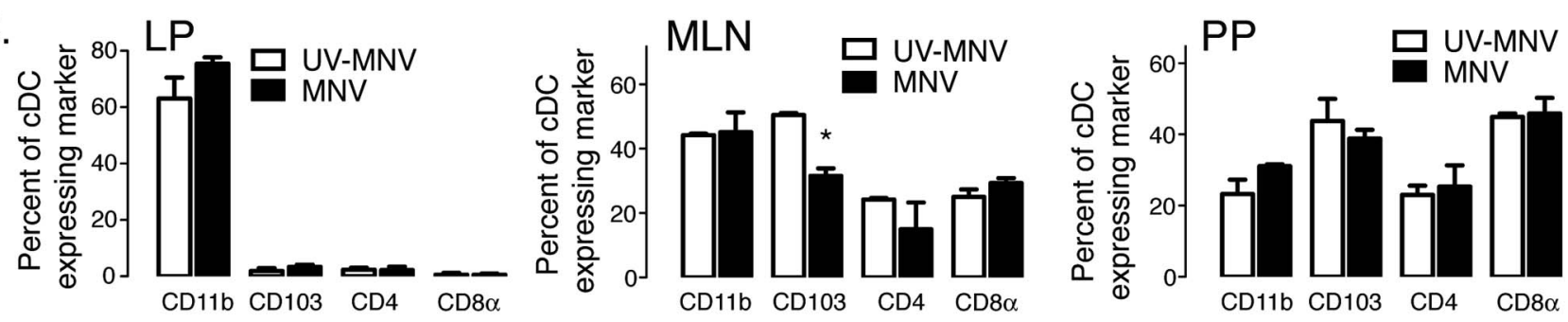

Fig. (2). Effect of MNV infection on cDCs from the Lamina Propria (LP), Peyer's Patch (PP), and mesenteric lymph node (MLN). (A) Gating strategy for flow cytometry. Single cell suspensions were made from lamina propria (LP), Peyer's Patches (PP) and mesenteric lymph nodes (MLN) and then stained with DAPI and various cell surface markers, as shown. Frequencies of CD11c-expressing cells (cDCs) or pDCA-1-expressing cells (pDCs) were analyzed by gating first on cellular events based on forward and side scatter, and then excluding DAPI positive cells (dead cells). Cells were then quantified as the percentage of cells in this gate that expressed the specific markers. (B) Time course of CD11c-expressing cDCs in different tissue compartments after MNV (filled circle), UV-MNV (open circle) or PBS (" $x$ ") inoculation. Data are shown as mean frequency \pm sem of cells at the indicated time p.i. and are from 3 independent experiments of $2-8$ mice/experiment. ${ }^{*} \mathrm{p}<0.05$ vs pre-inoculation value (day 0). Frequency of cells were determined as indicated in (A). (C) cDC subsets in LP, MLN or PP after UV-MNV or MNV inoculation. Sixteen hours after MNV or UV-MNV inoculation, the frequency of cDC's expressing $\mathrm{CD} 11 \mathrm{~b}, \mathrm{CD} 103$, CD4, or CD8 $\alpha$ were determined by flow cytometry. Data representative from 3 independent experiments of 2 mice per experiment. ${ }^{*} \mathrm{p}<0.05$ vs UV-MNV treated.

The PP, on the other hand, contained mainly CD103 and $\mathrm{CD} 8 \alpha$ expressing $\mathrm{cDC}$ subsets. Inoculation with MNV had no effect on the relative frequencies of these cDC subsets (Fig. 2C).

\section{Effect of MNV Infectionon pDC Tissue Kinetics}

Since pDCs play an important role in anti-viral immune responses by producing copious amounts of type I IFNs, we examined how MNV infection altered the frequency of pDCs 
A.
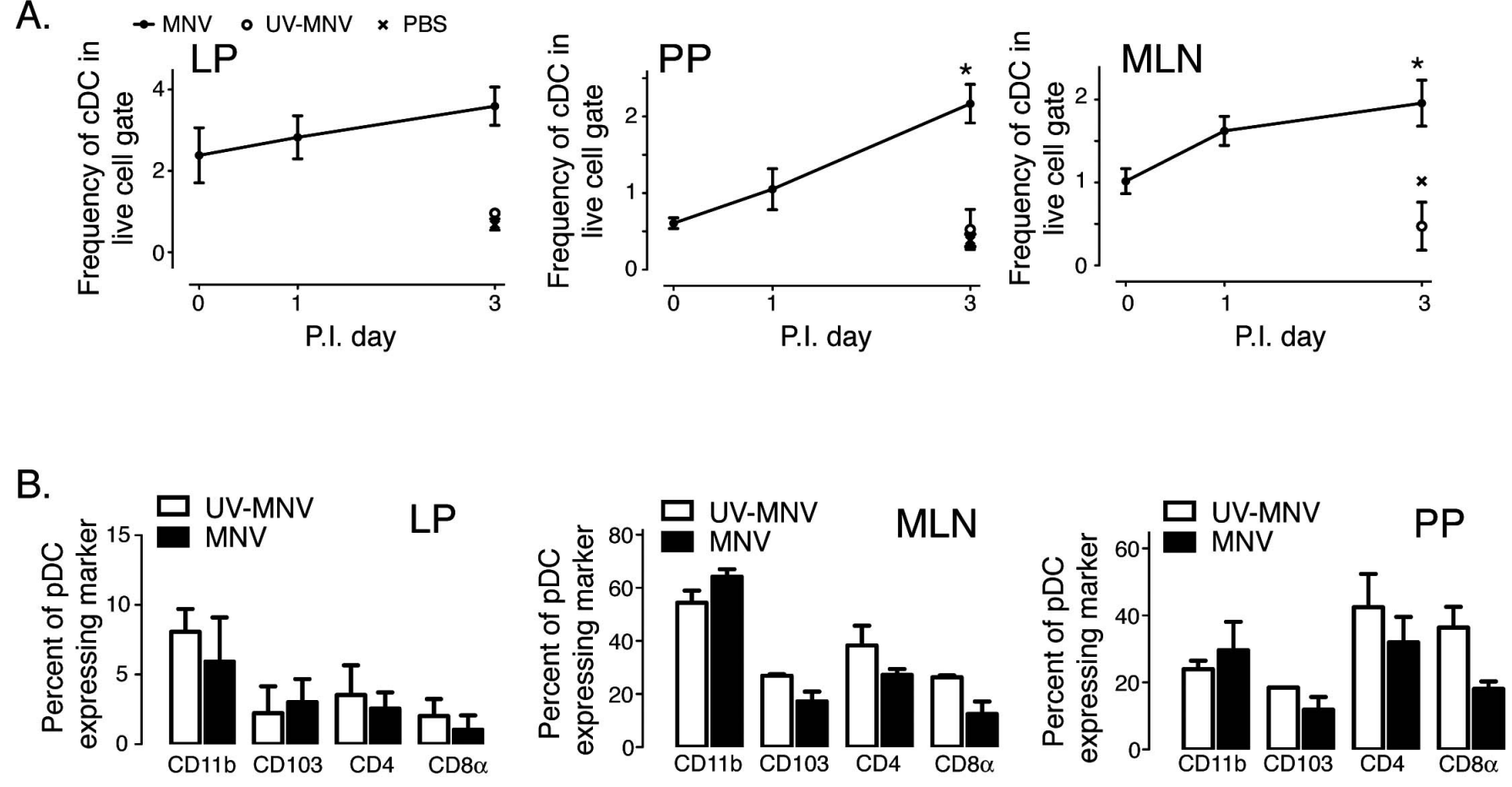

Fig. (3). MNV infection increases pDC frequencies in the PP and MLN but not in the LP. Data was collected and analyzed similar to Fig. 2. (A) Frequencies of pDCA-1 ${ }^{+}$cells in different tissues on the given days p.i. MNV (filled circle), UV-MNV (open circle), or PBS ("x"). ${ }^{*} \mathrm{p}<0.05$ vs day 0 (pre-inoculation value). Data compiled from 3 independent experiments of 2-8 mice per experiment. (B) Frequency of pDC subsets in LP, MLN and PP after UV-MNV or MNV inoculation. Plasmacytoid DC were identified as shown in Fig. 2C and the frequency of pDC expressing CD11b, CD103, CD4, or CD8 $\alpha$ determined 16 hours p.i. MNV or UV-MNV. Data are representative from 3 independent experiments of 2 mice per experiment. No differences were found to be statistically significant.

in the small intestine. We used the pDC specific surface marker PDCA-1 to identify mouse pDCs as we have done previously, and examined the frequency of pDCs in different tissues after MNV, UV-MNV, or PBS gavage (gating strategy shown in Fig. 2A) [16]. In contrast to what we saw with cDCs, the pDCs in both the PP and MLN increased significantly, while the frequencies of pDCs in the LP did not significantly change after MNV infection (Fig. 3A).

\section{MLN and PP pDC Subsets are Unaffected by MNV Infection}

Most pDC in the LP appeared to express none of the markers examined (Fig. 3B). MNV infection had no effect on $\mathrm{pDC}$ phenotype in the LP. In the MLN, however, phenotyping of pDCA- $1^{+}$cells showed that the majority of these cells also expressed CD11b (Fig. 3B); whereas, in the PP there was an even distribution of $\mathrm{pDC}$ subsets. Active viral infection had no significant effect on these frequencies in either of these organs.

\section{MNV Infection Leads to Transient Expression of FceRI on LP-cDCs}

We have previously shown that a respiratory infection with a single-stranded RNA virus, Sendai virus, induced expression of the high-affinity receptor for IgE (FceRI) on lung parenchyma cDCs [6]. Since MNV is also a singlestranded RNA virus, we examined expression of FceRI $\alpha$ and CD23 (the low-affinity IgE receptor) on LP-cDCs following inoculation with UV-MNV or MNV. While UV-MNV failed to induce expression at any time point (data not shown),
MNV infection led to a small but statistically significant increase in expression of both markers in LP-cDCs (Fig. 4A). In our respiratory viral model, we found that intranasal (i.n.) administration of ovalbumin (OVA) during the viral infection led to production of anti-ovalbumin IgE [32]. Since this response depended upon expression of cDC FceRI, we next examined whether a similar phenomenon could occur with MNV. In particular, we administered OVA by gavage 5 days post inoculation with either UV-MNV or MNV. As shown in Fig. (4B), 1 week after administration of the OVA only the MNV infected mice had increased levels of OVA specific IgE in their serum, suggesting that similar to the $\mathrm{SeV}$ model, exposure to a non-viral antigen during the antiMNV immune response is sufficient to drive IgE production against the non-viral antigen.

\section{DISCUSSION}

In this study we have examined the effect of a natural rodent pathogen, mouse norovirus (MNV), on dendritic cell subsets in the gastrointestinal tract. While several other studies have examined the effect of a double-stranded RNA virus (reovirus) on gastrointestinal dendritic cells, this is the first study that we are aware of to examine the effects of a single-stranded RNA virus on both $\mathrm{cDC}$ and $\mathrm{pDC}$ subsets in the gastrointestinal tract $[34,35]$. With inoculation of C57BL6 mice there is a rapid decrease in $\mathrm{cDC}$ numbers seen in the LP of the intestinal tract-particularly in the proximal $1 / 3$ of the small intestine, which is likely a direct result of the fact that MNV preferentially replicates in this part of the intestine [36]. This reduction in $\mathrm{cDC}$ in the primary target 
A.

FceRI

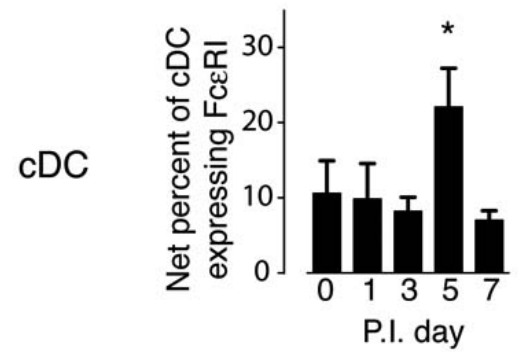

P.I. day

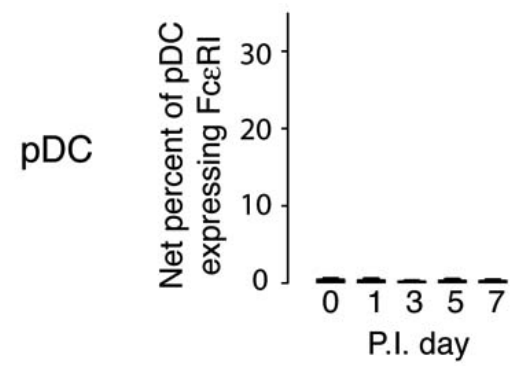

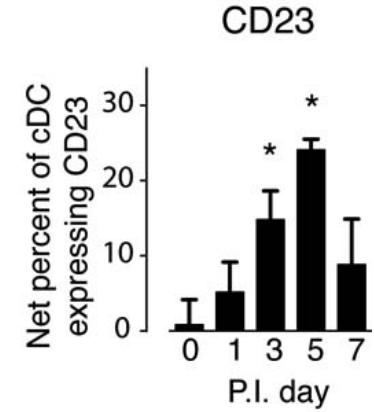

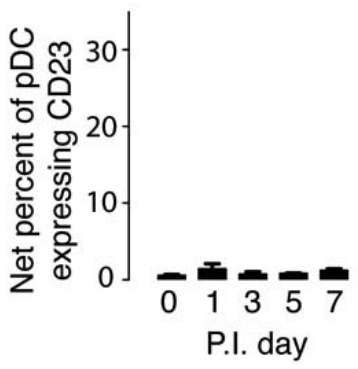

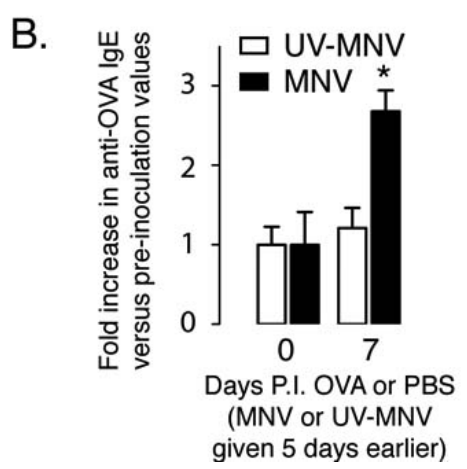

Fig. (4). MNV infection transiently increases receptors for IgE on LP-cDCs and drives a modest IgE response against non-viral antigens. (A) Expression of high-affinity (FceRI $\alpha$ ) and low-affinity (CD23) IgE receptors was determined on LP-cDCs and LP-pDCs by flow cytometry at the indicated days p.i. MNV. UV-MNV inoculation led to no increase in expression of these proteins at any of the time points examined (data not shown). Data shown as net mean \pm sem percent of LP-cDCs or LP-pDCs expressing the given marker, and are from 3 independent experiments, with 2 mice per experiment.*p $<0.05 v$ s pre-inoculation value (day 0). (B) Oral exposure to ovalbumin (OVA) during MNV infection is sufficient to induce production of anti-OVA IgE. Mice were inoculated with either MNV or UV-MNV and 5 days later gavaged with OVA or PBS. One-week later serum was collected and levels of anti-OVA IgE determined by ELISA. Data are presented as mean \pm sem fold increase of anti-OVA IgE over pre-inoculation values for mice given OVA or PBS at day 5 p.i. Data from 2 experiments with 3 mice per group. ${ }^{*} \mathrm{p}<0.05$ s both UV-MNV and baseline values.

tissue of the virus, which is not seen in the draining lymphoid tissue, is reminiscent of what we have seen with a respiratory infection with the single-stranded RNA virus, $\mathrm{SeV}$ [16]. The reduction in $\mathrm{cDC}$ numbers may be due to a combination of cellular death, as suggested by our in vitro culture (but clearly not proven, given the large variability in the PBS control group), and migration to draining lymph nodes; however, no evidence of increased numbers of $\mathrm{cDC}$ were found in the MLN or PP. These data would suggest that the decrease is due to death of cDC in the LP; alternatively, these cells could be trafficking to other sites, such as the spleen, which we did not examine. It is worthwhile noting that MNV has a tropism for macrophages and dendritic cells, and can replicate within these cells [37]. Therefore, we believe that much of the decrease in cell numbers seen in this model is likely secondary to a combination of cell death and migration.

With $\mathrm{SeV}$, we saw a robust accumulation of $\mathrm{pDC}$ in the lung within 1 day of viral inoculation. However, with MNV inoculation we found no increase in pDC in the LP (the target tissue of the viral infection), but we did see significant increases in both of the lymphoid organs studied (PP and MLN). This may relate to functionally different responses between the gastrointestinal and respiratory mucosa, or could reflect the relative difference in the severity of the infection following administration of $\mathrm{MNV}$, which we discuss in more detail below.

In terms of dynamics, while we examined the subsets of both $\mathrm{cDC}$ and $\mathrm{pDC}$ during an $\mathrm{MNV}$ infection, the only significant change was a reduction in $\mathrm{CD}_{103}{ }^{+} \mathrm{cDC}$ in the 
MLN. The relevance of these data are unclear given the relatively small change (from around $50 \%$ of $\mathrm{cDC}$ to approximately $30 \% \mathrm{cDC}$ ) in the frequency of these cells. Nonetheless, these data do suggest that inoculation with MNV does lead to an inflammatory response that changes the phenotype of the gastrointestinal dendritic cell network.

One of the most striking changes that occurred with respiratory infection with $\mathrm{SeV}$ was the induction of the highaffinity receptor for IgE (FceRI) on $\mathrm{cDC}$ and $\mathrm{pDC}$ [6]. While much less robust, inoculation with MNV was sufficient to drive a brief but statistically significant induction of FceRI on LP cDC. No expression of this receptor was noted on pDC, unlike what was seen in the respiratory model. Associated with expression of FceRI was the ability to drive an IgE mediated response against a non-viral antigen. This provides evidence that the development of atopic disease in the intestinal tract (food allergy, etc.) may relate to exposure to non-viral antigens during a viral infection. We have shown that in the respiratory tract a similar phenomenon occurs with $\mathrm{SeV}$ [32].

Interestingly, while the IgE response we saw was modest, it was generated with a single low dose $(120 \mu \mathrm{g})$ of OVAin other studies induction of anti-OVA IgE requires either a much higher dose (5 $\mathrm{mg}$ with an adjuvant) or repeated administration (9 weeks of daily administration of $100 \mu \mathrm{g}$ ) of OVA [38, 39]. Therefore, our study suggests that MNV infection itself is a very strong and effective adjuvant to drive this IgE mediated response even with a small antigen load. Whether differing doses of OVA would generate other effects we do not know, but it is interesting to speculate that a more robust IgE response would be seen with higher OVA doses.

While our study was done in a mouse model, it does have potential implications for human disease. In particular, our data suggest that even mild gastrointestinal tract viral infections are sufficient to drive the cDC - FceRI - IgE axis that leads to atopic disease. Given the rapid increase in food allergies seen in the westernized world, our data may provide a possible mechanism through which mild GI viral illnesses could translate into sensitization against a food allergen [40]. Unlike what we saw in the lung where we were able to link increased airway hyper-responsiveness with the development of anti-OVA IgE, it is important to remember that our studies only link sensitization to exposure during the GI viral infection - we have not studied clinical disease as a result of the anti-OVA IgE production. Therefore, it remains unclear if food allergy (a clinical disease) truly can result from food exposure during a gastrointestinal viral infection. Clearly, further studies in the human are needed to clarify this issue.

A major caveat to our studies is the fact that MNV causes a very weak infection, both clinically and in terms of the immunologic changes. In fact, many rodent facilities already have MNV infection in their colonies [12]. In our study, the mice came from Jackson Laboratories, where they did not have a prior exposure to MNV, so we believe the changes we have reported are due to the inflammatory response to an initial MNV exposure. Nonetheless, our data must be viewed in light of the fact that MNV infection really is not very virulent. In fact many of the differences seen between the $\mathrm{SeV}$ model and MNV may be explained by the fact that $\mathrm{SeV}$ can induce a fatal infection in wild-type mice, while MNV causes what is essentially a clinically silent infection that is rapidly cleared. However, it is also quite possible that the differences we have seen represent the diversity in the immune response to viral infection between mucosal sites.

\section{CONCLUSION}

In summary, our study documents a modest change in cDC with MNV inoculation, suggesting similar, but much less robust, changes to what is seen in the lungs with $\mathrm{SeV}$. These alterations include expression of FceRI on the LP cDC and the apparent ability to drive an IgE response to nonviral, ingested antigens. Taken together, this study provides additional support for the idea that atopic disease may be related to the antiviral immune response. Further studies will need to be undertaken with more virulent single-stranded RNA rodent gastrointestinal viruses to adequately determine changes in gastrointestinal dendritic cells and to test the hypothesis that food allergy is a result of a misdirected antiviral immune response.

\section{ACKNOWLEDGEMENTS}

We thank Dr. Herbert W. Virgin for providing MNVCW1 virus stock, and Dr. John Routes for the RAW 264.7 cell line. For technical support we thank Drs. Nita Salzman, Vera Tarakanova, and Xin Zhang, as well as Ms. Jennifer McGraw. We thank Dr. Dorothy Cheung for critical review of the manuscript. The National Institutes of Health (grant AI079905 and AI079905-02S1) and the Children's Research Institute of the Children's Hospital of Wisconsin supported this study.

\section{CONTRIBUTIONS}

XC, DL, DAH, DS, EJB, and SJZ were responsible for acquiring and analyzing data. $\mathrm{XC}$ and $\mathrm{MHG}$ were responsible for study design and drafting of the manuscript. MHG was also involved in analyzing data. All authors have read and approved the final version of the manuscript.

\section{CONFLICT OF INTEREST}

MHG has received research support from Genentech and the NIH. The other authors declare no competing financial interests.

\section{REFERENCES}

[1] Hall CB, Hall WJ, Gala CL, MaGill FB, Leddy JP. Long-term prospective study in children after respiratory syncytial virus infection. J Pediatr 1984; 105(3): 358-64.

[2] Sigurs N, Gustafsson PM, Bjarnason R, et al. Severe respiratory syncytial virus bronchiolitis in infancy and asthma and allergy at age 13. Am J Respir Crit Care Med 2005; 171(2):137-41.

[3] Sigurs N, Aljassim F, Kjellman B, et al. Asthma and allergy patterns over 18 years after severe RSV bronchiolitis in the first year of life. Thorax 2010; 65(12): 1045-52.

[4] Sly PD, Kusel M, Holt PG. Do early-life viral infections cause asthma? J Allergy Clin Immunol 2010; 125(6): 1202-5.

[5] Dahl ME, Dabbagh K, Liggitt D, Kim S, Lewis DB. Viral-induced $\mathrm{T}$ helper type 1 responses enhance allergic disease by effects on lung dendritic cells. Nat Immunol 2004; 5(3): 337-43.

[6] Grayson MH, Cheung D, Rohlfing MM, et al. Induction of highaffinity $\operatorname{IgE}$ receptor on lung dendritic cells during viral infection leads to mucous cell metaplasia. J Exp Med 2007; 204(11): 275969.

[7] Cheung DS, Ehlenbach SJ, Kitchens RT, et al. Cutting edge: $\mathrm{CD} 49 \mathrm{~d}+$ neutrophils induce FcepsilonRI expression on lung dendritic cells in a mouse model of postviral asthma. J Immunol 2010; 185(9): 4983-7. 
[8] Estes MK, Prasad BV, Atmar RL. Noroviruses everywhere: has something changed? Curr Opin Infect Dis 2006; 19(5): 467-74.

[9] Fankhauser RL, Noel JS, Monroe SS, Ando T, Glass RI. Molecular epidemiology of "Norwalk-like viruses" in outbreaks of gastroenteritis in the United States. J Infect Dis 1998; 178(6):15718 .

[10] Glass RI, Parashar UD, Estes MK. Norovirus gastroenteritis. N Engl J Med 2009; 361(18): 1776-85.

[11] Mead PS, Slutsker L, Dietz V, et al. Food-related illness and death in the United States. Emerg Infect Dis, 1999; 5(5): 607-25.

[12] Karst SM, Wobus CE, Lay M, Davidson J, Virgin HW. STAT1dependent innate immunity to a Norwalk-like virus. Science 2003; 299(5612): 1575-8.

[13] Chachu KA, LoBue AD, Strong DW, Baric RS, Virgin HW. Immune mechanisms responsible for vaccination against and clearance of mucosal and lymphatic norovirus infection. PLoS Pathog 2008; 4(12): e1000236.

[14] Coquerelle C, Moser M. DC subsets in positive and negative regulation of immunity. Immunol Rev 2010; 234(1): 317-34.

[15] Dzionek A, Fuchs A, Schmidt P, et al. BDCA-2, BDCA-3, and BDCA-4: three markers for distinct subsets of dendritic cells in human peripheral blood. J Immunol 2000; 165(11): 6037-46.

[16] Grayson MH, Ramos MS, Rohlfing MM, et al. Controls for lung dendritic cell maturation and migration during respiratory viral infection. J Immunol 2007; 179(3): 1438-48.

[17] Grayson MH. Lung dendritic cells and the inflammatory response. Ann Allergy Asthma Immunol 2006; 96(5): 643-51; quiz 652-3, 678.

[18] Maldonado-Lopez R, De Smedt T, Michel P, et al. CD8alpha+ and CD8alpha- subclasses of dendritic cells direct the development of distinct T helper cells in vivo. J Exp Med 1999; 189(3): 587-92.

[19] Grayson MH, Holtzman MJ. Emerging role of dendritic cells in respiratory viral infection. J Mol Med 2007; 85(10): 1057-68.

[20] Annacker O, Coombes JL, Malmstrom V, et al. Essential role for CD103 in the T cell-mediated regulation of experimental colitis. J Exp Med 2005; 202(8): 1051-61.

[21] Coombes JL, Siddiqui KR, Arancibia-Carcamo CV, et al. A functionally specialized population of mucosal CD103+ DCs induces Foxp3+ regulatory $\mathrm{T}$ cells via a TGF-beta and retinoic acid-dependent mechanism. J Exp Med 2007; 204(8): 1757-64.

[22] Laffont S, Siddiqui KR, Powrie F. Intestinal inflammation abrogates the tolerogenic properties of MLN CD103+ dendritic cells. Eur J Immunol 2010; 40(7): 1877-83.

[23] Vieira PL, de Jong EC, Wierenga EA, Kapsenberg ML, Kalinski P. Development of Th1-inducing capacity in myeloid dendritic cells requires environmental instruction. J Immunol 2000; 164(9): 450712.

[24] Edelson BT, Kc W, Juang R, et al. Peripheral CD103+ dendritic cells form a unified subset developmentally related to CD8alpha+ conventional dendritic cells. J Exp Med 2010; 207(4): 823-36.

[25] Facchetti F, Vermi W, Mason D, Colonna M. The plasmacytoid monocyte/interferon producing cells. Virchows Arch 2003; 443(6): 703-17.
[26] Fitzgerald-Bocarsly P. Human natural interferon-alpha producing cells. Pharmacol Ther 1993; 60(1): 39-62.

[27] Gilliet M, Cao W, Liu YJ. Plasmacytoid dendritic cells: sensing nucleic acids in viral infection and autoimmune diseases. Nat Rev Immunol 2008; 8(8): 594-606.

[28] Siegal FP, Kadowaki N, Shodell M, et al. The nature of the principal type 1 interferon-producing cells in human blood. Science 1999; 284(5421): 1835-7.

[29] O'Keeffe M, Hochrein H, Vremec D, et al. Mouse plasmacytoid cells: long-lived cells, heterogeneous in surface phenotype and function, that differentiate into $\mathrm{CD} 8(+)$ dendritic cells only after microbial stimulus. J Exp Med 2002; 196(10): 1307-19.

[30] Naik SH, Corcoran LM, Wu L. Development of murine plasmacytoid dendritic cell subsets. Immunol Cell Biol 2005; 83(5): 563-70.

[31] Yang GX, Lian ZX, Kikuchi K, et al. Plasmacytoid dendritic cells of different origins have distinct characteristics and function: studies of lymphoid progenitors $v s$ myeloid progenitors. J Immunol 2005; 175(11): 7281-7.

[32] Cheung DS, Ehlenbach SJ, Kitchens T, Riley DA, Grayson MH. Development of atopy by severe paramyxoviral infection in a mouse model. Ann Allergy Asthma Immunol 2010; 105(6): 437$443 \mathrm{e} 1$.

[33] Ballesteros-Tato A, Leon B, Lund FE, Randall TD. Temporal changes in dendritic cell subsets, cross-priming and costimulation via CD70 control CD8(+) $\mathrm{T}$ cell responses to influenza. Nat Immunol 2010; 11(3): 216-24.

[34] Johansson C, Wetzel JD, He J, Mikacenic C, Dermody TS, Kelsall BL. Type I interferons produced by hematopoietic cells protect mice against lethal infection by mammalian reovirus. J Exp Med 2007; 204(6): 1349-58.

[35] Fleeton MN, Contractor N, Leon F, Wetzel JD, Dermody TS, Kelsall BL. Peyer's patch dendritic cells process viral antigen from apoptotic epithelial cells in the intestine of reovirus-infected mice. J Exp Med 2004; 200(2): 235-45.

[36] Mumphrey SM, Changotra H, Moore TN, et al. Murine norovirus 1 infection is associated with histopathological changes in immunocompetent hosts, but clinical disease is prevented by STAT1-dependent interferon responses. J Virol 2007; 81(7): 325163.

[37] Wobus CE, Karst SM, Thackray LB, et al. Replication of norovirus in cell culture reveals a tropism for dendritic cells and macrophages. PLoS Biol 2004; 2(12): e432.

[38] Mathias CB, Hobson SA, Garcia-Lloret M, et al. IgE-mediated systemic anaphylaxis and impaired tolerance to food antigens in mice with enhanced IL-4 receptor signaling. J Allergy Clin Immunol 2011; 127(3): 795-805.

[39] Okunuki H, Teshima R, Sakushima J, et al. Induction of active systemic anaphylaxis by oral sensitization with ovalbumin in mastcell-deficient mice. Immunol Lett 2000; 74(3): 233-37.

[40] Boyce JA, Assa'ad A, Burks AW, et al. Guidelines for the diagnosis and management of food allergy in the United States: summary of the NIAID-sponsored expert panel report. J Allergy Clin Immunol 2010; 126(6): 1105-1118.

This is an open access article licensed under the terms of the Creative Commons Attribution Non-Commercial License (http://creativecommons.org/licenses/by$\mathrm{nc} / 3.0 /$ ) which permits unrestricted, non-commercial use, distribution and reproduction in any medium, provided the work is properly cited. 